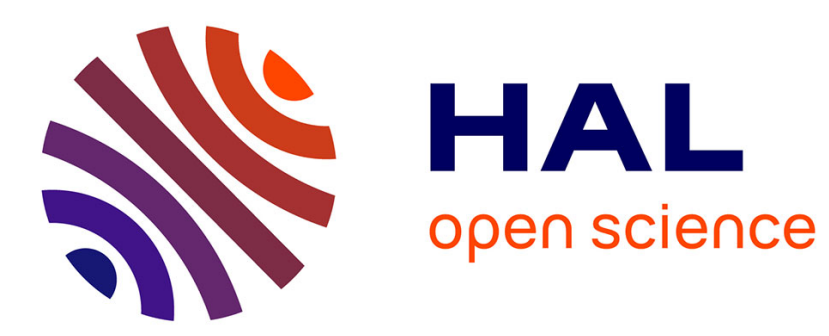

\title{
L'inflammasome NLRP3 : physiopathologie et application thérapeutique
}

Thomas Gicquel, Sacha Robert, Tatiana Victoni, Vincent Lagente

\section{To cite this version:}

Thomas Gicquel, Sacha Robert, Tatiana Victoni, Vincent Lagente. L'inflammasome NLRP3 : physiopathologie et application thérapeutique. La Presse Médicale, 2016, 45 (4, part 1), pp.438-446. 10.1016/j.lpm.2016.01.006 . hal-01274225

HAL Id: hal-01274225

https://hal-univ-rennes1.archives-ouvertes.fr/hal-01274225

Submitted on 29 Sep 2016

HAL is a multi-disciplinary open access archive for the deposit and dissemination of scientific research documents, whether they are published or not. The documents may come from teaching and research institutions in France or abroad, or from public or private research centers.
L'archive ouverte pluridisciplinaire HAL, est destinée au dépôt et à la diffusion de documents scientifiques de niveau recherche, publiés ou non, émanant des établissements d'enseignement et de recherche français ou étrangers, des laboratoires publics ou privés. 


\section{L'inflammasome NLRP3: Physiopathologie et application thérapeutique}

\section{The NLRP3 inflammasome : Physiopathology and therapeutic application}

Thomas Gicquel $^{1,2,3}$, Sacha Robert ${ }^{2,3}$, Tatiana Victoni ${ }^{2,3}$, Vincent Lagente ${ }^{2,3}$

1. CHU Rennes, Laboratoire de toxicologie biologique et médico-légale, F-35033 Rennes, France.

2. Univ. Rennes 1, Faculté de pharmacie, F-35043 Rennes, France

3. INSERM, UMR991 «Foie, Métabolismes et Cancer» F-35043 Rennes, France

Correspondance :

Thomas Gicquel, Laboratoire de toxicologie biologique et médico-légale, CHU Pontchaillou, F-35033 Rennes, France. thomas.gicquel@chu-rennes.fr 


\section{Points essentiels}

L'immunité innée constitue la première ligne de défense de l'organisme contre les pathogènes.

La réponse inflammatoire permet de lutter contre le «non-soi» comme les organismes infectieux ou les particules exogènes.

L'inflammasome est un complexe multiprotéique intracytoplasmique activé par des stress cellulaires ou des infections et sont responsables de la libération de cytokines proinflammatoires, notamment l'IL-1 $\beta$.

L'inflammasome NLRP3, le plus étudié des inflammasomes, est impliqué dans des pathologies inflammatoires telles que la maladie de Crohn, la polyarthrite rhumatoïde ou l'arthrite goutteuse.

Les CAPS (Syndromes périodiques associés à la cryopyrine) sont des pathologies spécifiques de l'inflammasome NLRP3.

Le canakinumab (Ilaris ${ }^{\circledR}$ ) est la seule spécialité ayant eu l'autorisation de mise sur le marché (AMM) dans le traitement des CAPS.

Les biothérapies ciblant l'inflammasome NLRP3 et l'IL-1 $\beta$ peuvent représenter de nouvelles armes thérapeutiques anti-inflammatoires.

\section{Key points}

The innate immune system constitutes the first line of host defense against pathogens.

« Non-self», such as exogenous particles or pathogens, triggers an inflammatory response.

Inflammasomes are molecular platforms activated upon cellular infection or stress that trigger the maturation of proinflammatory cytokines such as IL-1 $\beta$.

Activation of the NLRP3-inflammasome pathway, the most extensively studied, appears to be the corner stone of many inflammatory diseases, including Crohn's disease, rheumatoid arthritis and gout.

CAPS (Cryopyrine-associated periodic syndromes) are NLRP3 inflammasome-associated diseases.

Canakinumab (Ilaris $\left.{ }^{\circledR}\right)$ is the only drug approved for CAPS treatment in France.

Targeted therapy against NLRP3-inflammasome and IL-1 $\beta$ might be the new antiinflammatory drugs. 


\section{Glossaire}

\begin{tabular}{|c|c|}
\hline AIM2 & Absent in melanoma 2 \\
\hline AINS & Anti-inflammatoire non stéroïdien \\
\hline AJIS & Arthrite juvénile idiopathique systémique \\
\hline ALR & AIM2-like receptor \\
\hline AMM & Autorisation de mise sur le marché \\
\hline AMPc & Adenosine monophosphate cyclique \\
\hline ASC & Apoptosis-associated speck-like protein containing a CARD domain \\
\hline ATP & Adénosine triphosphate \\
\hline CAPS & Cryopyrin-associated periodic syndrome \\
\hline CARD & Caspase recruitment domain \\
\hline CINCA & Chronic infantile neurological cutaneous articular syndrome \\
\hline COX-2 & Cyclo-oxygenase de type 2 \\
\hline CRP & Protéine $\mathrm{C}$ réactive \\
\hline DAMP & Danger associated molecular pattern \\
\hline FCAS & Familial cold autoinflammatory syndrome \\
\hline FMF & Fièvre médittéranéenne familiale \\
\hline ICE & Interleukine- $1 \beta$ converting enzyme \\
\hline IFN & Interféron \\
\hline IL & Interleukine \\
\hline IL-1Ra & Interleukine 1 Receptor antagonist \\
\hline LPS & Lipopolysaccharide \\
\hline LRR & Leucine rich repeat \\
\hline MWS & Muckle-Wells syndrom \\
\hline $\mathbf{N F}-\kappa \mathrm{B}$ & Nuclear factor $\kappa \mathrm{B}$ \\
\hline NLR & NOD-like receptor \\
\hline NLRC & NOD-, LRR-, and CARD domain-containing \\
\hline NLRP & NOD-, LRR-, and pyrin domain-containing \\
\hline NO & Monoxyde d'azote \\
\hline NOD & Nucleotide-binding oligomerization domain \\
\hline NOMID & Neonatal onset multi-systemic inflammatory disease \\
\hline PAMP & Pathogen associated molecular pattern \\
\hline PGE2 & Prostaglandine E2 \\
\hline PRR & Pattern Recognition Receptors \\
\hline PYD & Pyrin domain \\
\hline ROS & Reactive oxygen species \\
\hline TIR & Toll-Interleukin-1 receptor \\
\hline TLR & Toll Like Receptor \\
\hline TNF & Tumor necrosis factor \\
\hline
\end{tabular}


L'inflammasome a été décrit comme une plate-forme multiprotéique permettant la maturation de cytokines pro-inflammatoires comme l'interleukine-1 $\beta$ (IL-1 $\beta$ ). Actuellement, l'inflammasome NLRP3 est le plus étudié et celui-ci est impliqué dans de nombreuses pathologies inflammatoires. Il peut être activé par une multitude de signaux d'origine pathogène ou non et permet le clivage de la pro-IL-1 $\beta$ en IL-1 $\beta$ active. Le mécanisme moléculaire exact de l'activation de ce complexe est encore mal connu et une meilleure compréhension de cette voie de signalisation pourrait permettre une application dans le traitement des pathologies inflammatoires.

\section{Immunité innée et inflammation}

L'immunité innée est un système universel de défense contre les pathogènes. L'identification de structures moléculaires par les récepteurs de l'immunité permet la reconnaissance du « soi » et du « non soi ». La réponse inflammatoire fait partie de l'immunité innée et permet de lutter contre le «non soi » comme des organismes infectieux ou des particules exogènes. Chez les mammifères, l'inflammation va dans un premier temps être initiée par l'activation des mastocytes, polynucléaires, monocytes et macrophages [1]. Cette réponse va se caractériser par la sécrétion locale de cytokines pro-inflammatoires, comme le Tumor necrosis factor- $\alpha$ (TNF- $\alpha$ ) et l'interleukine-1 $\beta$ (IL-1 $\beta$ ), mais aussi de chimiokines facilitant le recrutement de cellules immunitaires au site d'infection. L'activation des récepteurs de l'immunité va permettre le déclenchement de nombreux mécanismes comme la cascade du complément, la phagocytose ou l'activation de voies de signalisation pro-inflammatoires [1]. Une étape clé de l'immunité innée et de la réponse inflammatoire est la reconnaissance des composés d'origine pathogène, appelés Pathogen-associated molecular patterns (PAMPs) ou des molécules de dangers, les Danger-associated molecular patterns (DAMPs) par les récepteurs de l'immunité innée, les Pattern recognition receptors (PRRs). Ces récepteurs des cellules de l'immunité innée sont répartis selon leur localisation, les NOD-like receptors (NLRs) sont intracytoplasmique alors que les Toll-like receptors (TLRs) seront transmembranaires [2]. 


\section{Les TLRs}

Les récepteurs Toll ont été mis en évidence chez la mouche drosophile (Drosophila melanogaster) comme des récepteurs de l'immunité permettant de lutter contre les infections fongiques [3]. Ce même type de récepteur a également été trouvé chez les mammifères. Etant très conservés au cours de l'évolution, ils présentent les mêmes spécificités structurales que la molécule Toll de la drosophile. Le premier homologue de ces récepteurs a été décrit par Medzhitov et a été mis en évidence comme étant impliqué dans l'induction des gènes entrainant une réponse inflammatoire et correspondait au TLR4. Les TLRs sont des protéines situées sur la membrane plasmique ou la membrane des endosomes. Ces récepteurs sont impliqués dans la reconnaissance des pathogènes et jouent un rôle primordial dans l'immunité innée. La fixation des PAMPs provenant de bactéries, champignons, virus ou de protozoaires sur les récepteurs de l'immunité innée va entrainer une réponse inflammatoire [1]. Chez l'homme, dix TLRs différents (TLR1 à TLR10) ont été identifiés et sont localisés sur de nombreux types cellulaires. Les TLRs sont principalement retrouvés dans les cellules immunitaires comme les macrophages, les cellules dendritiques, les polynucléaires ou les lymphocytes mais également sur des cellules en contact avec le milieu extérieur comme les cellules pulmonaires ou les cellules épithéliales. La fonction principale des TLRs est la reconnaissance des pathogènes et l'activation des cellules qui les portent [4]. Ces protéines transmembranaires de type I sont toutes structurées en trois domaines. Un domaine extracellulaire Leucine-rich repeat (LRR), qui permet la reconnaissance des composés microbiens; un domaine transmembranaire et un domaine intracytoplasmique Toll/IL-1 Receptor (TIR) permettant la transduction du signal activateur. Le domaine TIR des TLRs, présente une grande similarité avec celui des récepteurs de la famille de l'IL-1. Cependant, les domaines extracellulaires sont différents de ceux des TLRs car ils présentent un domaine LRR permettant la reconnaissance des composés bactériens, alors que le récepteur à l'IL-1 possède un domaine immunoglobuline-like [5].

Les TLRs peuvent être localisés à la surface de la cellule (comme TLR1, 2, 4, 5 et 6) ce qui permet la détection de composés microbiens extracellulaires ou bien être localisés dans les endosomes ou lysosomes des cellules (comme les TLRs 3, 7,8 et 9) qui reconnaissent des acides nucléiques exogènes. Ainsi chaque TLR possède ses propres ligands activateurs, par exemple le Lipopolysaccharide (LPS), une endotoxine de la paroi des bactéries gram négatif, 
qui est spécifiquement reconnu par le TLR4. Le LPS est bien connu pour stimuler les réponses inflammatoires de l'organisme et peut être responsable de chocs septiques [6].

\section{Les NLRs}

Contrairement aux TLRs, les NLRs sont des PRRs cytoplasmiques qui ne contiennent pas de domaine TIR. Vingt-deux NLRs ont été identifiés chez l'homme par leur domaine structural et leurs relations phylogénétiques [7]. Ceux-ci ont été subdivisés en 5 catégories : NLRA, NLRB, NLRC, NLRP, et NLRX. Ils possèdent tous un domaine NACHT central et un domaine LRR au niveau C-terminal, excepté pour NLRP10. Ce sont leurs domaines Nterminaux qui permettent leur classification [8]. La famille de NLRs la plus étudiée est celle des NLRPs (NLRP1-14). En effet certains membres de cette famille peuvent former des complexes oligomériques, les inflammasomes après activation par des perturbations cytosoliques [9]. Certains NLRs, comme NLRC1, NLRC2, NLRC5 ou NLRX1 sont incapables de former des inflammasomes, pourtant ceux-ci jouent un rôle dans la réponse inflammatoire [10]. Parmi les 22 NLRs décrits chez l'homme, seuls NLRP1, NLRP3, NLRC4 et AIM2 peuvent former des inflammasomes.

\section{L'inflammasome}

L'inflammasome est décrit comme une plate-forme intra-cytoplasmique de haut poids moléculaire (de l'ordre de $10^{6} \mathrm{Da}$ ) permettant la maturation de cytokines pro-inflammatoires comme l'IL-1 $\beta$ [11]. Les inflammasomes sont des complexes protéiques intracellulaires de l'immunité innée constitués d'un NLR ou d'un ALR (AIM2-like receptor), d'une protéine adaptatrice et d'une cysteine protéase [12].

\section{Inflammasome NLRP3}

La protéine NLR la plus étudiée est NLRP3, anciennement appelée NALP3 ou Cryopyrine. Comme toutes les NLRPs, exceptées NLRP1 et NLRP10, la protéine NLRP3 est constituée d'un domaine PYD en position N-terminale, un domaine NACHT et un domaine LRR en position C-terminale. Cette protéine est un élément d'un complexe macromoléculaire, l'inflammasome NLRP3[13]. Le mieux caractérisé des inflammasomes est l'inflammasome NLRP3. Cet inflammasome est très exprimé dans les cellules myéloïdes comme les monocytes ou les macrophages [14]. L'inflammasome NLRP3 contient une protéine adaptatrice ASC (Adaptor protein apoptosis-associated speck like protein containing a CARD), la pro-caspase-1 et la protéine NLRP3 (Fig. 1). 
Après activation, NLRP3 s'oligomérise via des interactions homotypiques entre les domaines NACHT et présente son domaine Pyrin au domaine Pyrin de la protéine accessoire ASC, indispensable à l'activation de l'inflammasome [15]. ASC interagit via son domaine CARD avec le domaine CARD de la pro-caspase-1. Le recrutement de la pro-caspase-1 entraine l'oligomérisation de l'inflammasome et l'activation de celui-ci. Anciennement appelée IL-1 $\beta$ converting-enzyme (ICE), la caspase-1 est synthétisée sous forme inactive, la pro-caspase-1 qui nécessite une activation par un inflammasome pour pouvoir cliver la pro-IL-1 $\beta$ en IL-1 $\beta$ active [16,17]. Lors de l'assemblage de l'inflammasome NLRP3, la caspase-1 est activée par formation d'un hétérotétramère $\alpha 2 \beta 2$ constitué de deux protéines $\mathrm{p} 20$, contenant le site actif, et de deux sous-unités p10 [18]. Parmi les différentes caspases, seule la caspase-1 est capable de cliver la pro-IL-1 $\beta$. Cette cystéine protéase est aussi capable de cliver d'autres cytokines pro-inflammatoires comme la pro-IL-18 et peut également jouer un rôle dans la mort cellulaire en réponse aux agents pathogènes, dans le cas de la pyroptose [19]. Une fois activée, la caspase-1 va cliver le pro-peptide au niveau du résidu acide aspartique en position 116, produisant ainsi l'IL-1 $\beta$ mature, biologiquement active, qui sera libérée dans l'espace extracellulaire.

\section{IL-1及}

L'IL-1 $\beta$ est une cytokine qui joue un rôle majeur dans la réponse inflammatoire, infectieuse, les maladies dégénératives et la réparation tissulaire [20]. Cette cytokine pro-inflammatoire est principalement produite lors d'activation de cellules de l'immunité innée comme les monocytes ou les macrophages cependant les lymphocytes ou des cellules non immunitaires comme les kératinocytes semblent capables de libérer de 1'IL-1 $\beta$ [21,22]. Administrée par voie intraveineuse, l'IL-1 $\beta$ induit chez l'homme une hypotension, une anorexie et de la fièvre. Ce puissant pyrogène d'origine endogène va initier et amplifier de nombreuses réponses immunitaires et inflammatoires. Ainsi, l'IL-1 $\beta$ va augmenter la survie et les fonctions effectrices des cellules immunitaires comme les lymphocytes, les monocytes et les macrophages. L'IL-1 $\beta$ va également induire l'expression de gènes pro-inflammatoires comme la Prostaglandine E2 (PGE2), la Cyclo-oxygenase de type 2 (COX-2), l'IL-6 ou le TNF- $\alpha$ déclenchant une réponse immunitaire de type Th1 [23]. L'IL-1 $\beta$ possède deux récepteurs IL1R1 et IL-1R2 mais seule la fixation à 1'IL-1R1 membranaire entraine une cascade de signalisation. La fixation de l'IL-1 $\beta$ sur l'IL-1R1 entraine une réponse spécifique pour chaque type cellulaire. Cette cytokine va induire l'expression des protéines de l'inflammation par les 
hépatocytes, comme la protéine $\mathrm{C}$ réactive (CRP). L'IL-1 $\beta$ induit également la synthèse de chimiokines et de molécules d'adhérence par les cellules endothéliales entrainant ainsi le recrutement des leucocytes ou le déclenchement de la fièvre au niveau cérébral [23,24]. La production de l'IL-1 $\beta$ est très régulée au niveau de son expression, sa transcription et sa libération [25]. Trois étapes sont nécessaires à sa libération dans le milieu extracellulaire. Tout d'abord la production de son précurseur inactif, la pro-IL-1 $\beta$ puis le clivage de la pro-IL$1 \beta$ en IL-1 $\beta$ mature biologiquement active et enfin sa libération. Ces multiples mécanismes semblent avoir été développés pour assurer une protection de l'organisme vis-à-vis des propriétés pro-inflammatoires de cette puissante cytokine [15]. La transcription de la pro-IL$1 \beta$ doit être induite par un signal pro-inflammatoire car elle n'est pas exprimée de façon constitutive par les cellules [26]. Son expression est induite dans un contexte inflammatoire comme dans les monocytes ou macrophages activés. L'induction de cette transcription peut se faire via les TLRs ou induite par la fixation de cytokines endogènes sur leurs récepteurs

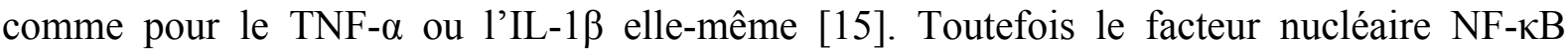
semble indispensable à l'induction de l'expression de la pro-IL-1 $\beta$ [14]. Cependant une induction de l'expression des gènes ou de la synthèse de la pro-IL-1 $\beta$ ne signifie pas forcément une augmentation de la production d'IL-1 $\beta$ biologiquement active [15]. En effet, en l'absence d'un clivage rapide de la pro-IL-1 $\beta$ par l'inflammasome NLRP3, la libération d'IL$1 \beta$ dans le milieu reste très modérée [27]. La libération d'IL-1 $\beta$ se fait de façon non conventionnelle, mais le mécanisme physiologique exact reste encore mal connu et dépend du type cellulaire. La libération peut se faire par formation de corps multivésiculaires et d'exosomes, par exocytose de lysosomes, par libération de microvésicules ou par des transporteurs membranaires spécifiques [28]. Ainsi, il est difficile de conclure sur la voie de libération principale, mais il semble que l'IL-1 $\beta$ peut être libérée par deux ou trois façons différentes, en parallèle.

\section{Activation de l’inflammasome NLRP3}

Les inflammasomes possèdent de nombreux activateurs, d'origine pathogènes, comme les PAMPs ou des signaux de danger, comme les DAMPs. Ainsi, l'inflammasome NLRP1 peut par exemple être activé par la toxine de Bacillus anthracis. NLRC4 peut être activé par la flagelline des bactéries à Gram négatif comme Legionella pneumophila, Salmonella typhimurium, Shigella flexneri, ou Pseudomonas aeruginosa [29]. L'inflammasome NLRP3 possède quant à lui de très nombreux activateurs d'origine très variée dont certains impliqués dans des pathologies (Tableau I). 
Les DAMPs activateurs de l'inflammasome NLRP3 peuvent être libérés suite à une infection, un stress métabolique ou une lésion cellulaire. Par exemple, l'ATP extracellulaire libéré est connu pour activer l'inflammasome NLRP3 via les récepteurs purinergiques, notamment P2X7R [31,32], tout comme les cristaux d'acide urique [33,34]. Etant donné l'hétérogénéité des molécules pouvant activer l'inflammasome NLRP3, une interaction directe entre ces molécules et NLRP3 parait très peu probable. Ainsi, de nombreux mécanismes sont proposés pour l'activation de cet inflammasome. Trois modèles sont majoritairement exprimés dans la littérature. L'activation de l'inflammasome NLRP3 par un efflux potassique $\left(\mathrm{K}^{+}\right)$, par les espèces réactives de l'oxygène (ROS) ou par la rupture des lysosomes [18,34,35]. De nombreuses observations confirment le rôle des flux ioniques dans l'activation de l'inflammasome car tous les activateurs de NLRP3 induisent un flux de $\mathrm{K}^{+}$, de même l'inflammasome NLRP3 semble nécessiter un assemblage sous forme de multimère pour devenir actif [36]. Un autre mode d'activation de l'inflammasome NLRP3 est la rupture du lysosome par les activateurs de grande taille comme les cristaux de silice, d'aluminium ou d'acide urique [37-40]. Il semblait que l'activation de l'inflammasome NLRP3 par les cristaux nécessitait la phagocytose, pourtant des cellules incapables de phagocyter comme les kératinocytes peuvent activer l'inflammasome NLRP3 et sécréter de l'IL-1 $\beta$ en réponse à des particules [41]. Un mécanisme de «phagocytose frustrée » des particules et des cristaux de grande taille a donc été proposé [40]. Ce mécanisme met en jeu le cytosquelette, en effet l'inhibition des filaments du cytosquelette par des molécules comme la colchicine ou la cytochalasine D diminue la libération d'IL-1 $\beta$ [38] Après internalisation des cristaux, une clairance inefficace va entrainer la production de ROS [42], un efflux de potassium [43], une déstabilisation du phagosome et une rupture du lysosome. Ceci va entrainer le relargage de son contenu dans le cytoplasme dont des protéases lysosomales comme la cathepsine B qui va entrainer l'activation de la caspase-1 [39] (Figure 2).

\section{Exemple d'activation de l'inflammasome NLRP3 par les cristaux d'acide urique}

L'acide urique est un catabolite des purines qui va précipiter sous forme de cristaux d'urate de sodium (Monosodium urate ou MSU) comme dans l'arthrite goutteuse et induire ainsi une inflammation [44,45]. L'acide urique va pouvoir être relargué par les cellules comme un signal de danger et va précipiter en présence de fortes concentrations de sodium présent dans l'environnement extracellulaire [45-47]. Il a été montré que le CD14 et les TLRs, comme TLR2 et TLR4, contribuaient à la production de cytokines en réponse aux cristaux d'acide urique $[48,49]$. Cependant, les TLRs ne détectent pas directement les cristaux et ne sont pas 
indispensables à la libération d'IL-1 $\beta$ en réponse au MSU [50]. En effet, le MSU semble être reconnu directement par le cholestérol de la membrane des phagocytes, sans intervention de récepteurs de surface. Ceci va être suffisant pour entrainer une cascade de signalisation intracellulaire [51]. De plus, il a été montré que le MSU, comme d'autres cristaux pouvait entrainer une libération d'ATP endogène [52]. Ceci laisse imaginer qu'une activation autocrine des récepteurs purinergiques en réponse à l'ATP endogène est possible. Ces différents mécanismes induiraient l'activation de l'inflammasome NLRP3 en réponse au MSU [33]. Ces dernières années, de nombreux autres mécanismes ont été mis en évidence ce qui rend d'autant plus complexe la compréhension de cette voie d'activation. Une régulation négative de l'inflammasome NLRP3 est indispensable à la résolution de l'inflammation. Ainsi, plusieurs mécanismes concourent à l'inhibition de celui-ci. En effet il a été montré que la fixation de l'IFN- $\alpha$, l'IFN- $\beta$ ou IL-10 sur leurs récepteurs, un microARN endogène miR223, l'AMP cyclique (AMPc) ou le NO (Nitric Oxyde) inhiberait le clivage de la pro-IL-1 $\beta$ par l'inflammasome NLRP3 [26,53]. Le mode d'activation de l'inflammasome NLRP3 ne semble pas exclusif. Pour avoir une réponse inflammatoire complète, plusieurs mécanismes doivent être mis en jeu simultanément. Il est donc primordial de comprendre ce mode d'activation car cette plateforme pro-inflammatoire est impliquée dans de nombreuses pathologies.

\section{Pathologies associées à l'inflammasome NLRP3}

L'inflammasome NLRP3 est impliqué dans de très nombreuses pathologies inflammatoires notamment la maladie de Crohn, la polyarthrite rhumatoïde ou l'arthrite goutteuse [45, 5456]. Il existe également des pathologies génétiques spécifiques de l'inflammasome NLRP3 appelées inflammasomopathies ou cryopyrinopathies.

\section{Les cryopyrinopathies}

Les syndromes périodiques associés à la cryopyrine (CAPS) sont des pathologies autoinflammatoires héréditaires. Trois maladies pédiatriques composent les CAPS : l'urticaire familial au froid (FCAS - Familial cold autoinflammatory syndrome), le syndrome de Muckle-Wells (MWS) et le syndrome NOMID/CINCA (Neonatal onset multi-systemic inflammatory disease / chronic infantile neurological cutaneous articular syndrom) [55]. Cliniquement, ces inflammasomopathies intrinsèques sont caractérisées par des symptômes 
généraux constants tels que des fièvres récurrentes d'origine non pathogène, des frissons et une fatigue intense. Les manifestations cliniques apparaissent le plus souvent en période néonatale et atteignent notamment la peau, les articulations ou le système nerveux central. Différents niveaux de gravité sont observés concernant les douleurs articulaires, les rashs cutanés, les atteintes ORL et les symptômes oculaires [57]. Dans le cas du NOMID/CINCA, la pathologie la plus sévère, un retard mental, un déficit auditif progressif, une dysmorphie faciale et une amylose sont les plus souvent retrouvés [58].

La découverte de ces maladies rares a permis de mieux comprendre le rôle de l'inflammasome NLRP3 dans la libération d'IL-1 $\beta$. Des mutations sur le gène codant pour la protéine NLRP3 (situé en 1q44 sur la portion courte du chromosome 1) et plus particulièrement le domaine NACHT sont responsables d'une activation constitutive de l'inflammasome NLRP3 dans les CAPS [57]. Une dérégulation de l'activation de l'inflammasome par synthèse d'un récepteur NLRP3 spontanément activé va provoquer une hyperproduction d'IL-1 $\beta$ et un état hyperinflammatoire récurrent, se traduisant par des symptômes allant de la simple éruption cutanée jusqu'à la mise en jeu du pronostic vital. Il a été montré une importante sécrétion basale d'IL-1 $\beta$ mature par les monocytes et macrophages de ces patients en l'absence de toute stimulation [59]. L'auto-induction de libération d'IL-1 $\beta$ est probablement le mécanisme majeur de nombreuses maladies inflammatoires. Ainsi, la cascade d'activation de l'inflammasome NLRP3 représente une cible thérapeutique importante dans le traitement de ces pathologies chroniques.

Il a récemment été mis en évidence que l'inflammasome NLRP3 était impliqué dans des pathologies sémiologiquement proches des cryopyrinopathies, telles que la maladie de Still ou le syndrome de Schnitzler $[55,60]$. Ces pathologies fébriles multisystémiques font partis des formes non mendéliennes des syndromes auto-inflammatoires [61].

Cependant, toutes les cryopyrinopathies ne sont pas dues à une mutation du gène codant pour la protéine NLRP3 [62]. Ainsi, l'inflammasome NLRP3 est également impliqué dans la fièvre méditerranéenne familiale (FMF), la maladie auto-inflammatoire héréditaire la plus fréquente au monde [63]. Cette inflammasomopathie extrinsèque se développe dès l'enfance et apparait généralement par crises sous forme de pics fébriles et de péritonites mais les symptômes articulaires et cutanés sont plus rares que dans les CAPS. La FMF est liée à une mutation du gène $M F E V$ codant pour la pyrine et entraine ainsi une dérégulation de la libération d'IL-1 $\beta$. 
Cependant, le rôle exact de la pyrine dans la physiopathologie de la FMF reste mal connu $[63,64]$.

De plus, il a été montré que des mutations de NLRP12 et de NLRC4 entrainaient des syndromes auto-inflammatoires aux symptômes semblables à ceux des cryopyrinopathies [65-67]. Ceci semble être dû à une hyperactivation de la caspase-1 entrainant une surproduction d'IL-1 $\beta$ [67].

\section{Traitement des cryopyrinopathies}

A l'heure actuelle, Ilaris ${ }^{\circledR}$ est la seule spécialité ayant l'autorisation de mise sur le marché (AMM) dans le traitement des cryopyrinopathies en France depuis 2009. Cependant d'autres traitements, ciblant la cytokine pro-inflammatoires IL-1 $\beta$, peuvent être utilisés hors AMM.

\section{Canakinumab}

Le canakinumab (Ilaris ${ }^{\circledR}$ ) est un anticorps monoclonal de type IgG1 dirigé spécifiquement contre l'IL-1 $\beta$ humaine [68]. Cet anticorps se lie avec une haute affinité à l'IL-1ß humaine et neutralise son activité biologique en inhibant son interaction avec les récepteurs à l'IL-1 et limite ainsi les effets pro-inflammatoires de l'IL-1 $\beta$ [69]. Le canakinumab est la seule molécule possédant l'AMM dans le traitement des CAPS. La spécialité Ilaris ${ }^{\circledR}$ est indiquée chez les adultes, mais également chez les enfants de plus de 2 ans. La posologie d'administration est d'une injection sous cutanée toutes les 8 semaines. Depuis 2013, cette spécialité est également indiquée dans le traitement d'autres pathologies telles que l'arthrite juvénile idiopathique systémique (AJIS) ou l'arthrite goutteuse. Le traitement par canakinumab a montré une grande efficacité chez les patients de plus de 2 ans atteints de l'AJIS [70]. Une amélioration rapide et prolongée des manifestations aussi bien articulaires que systémiques a été observée avec une réduction significative du nombre d'articulations inflammatoires, la résolution rapide de la fièvre et une réduction des marqueurs biologiques de la phase aiguë de l'inflammation chez la majorité des patients [71]. Dans le cas de l'arthrite goutteuse, l'activation de l'inflammasome NLRP3 et donc la libération d'IL-1 $\beta$ est due à la précipitation de l'acide urique sous forme de cristaux. L'activation des macrophages et la production concomitante d'IL-1ß active entraînent une réponse inflammatoire aiguë douloureuse [72]. Ainsi, le canakinumab a montré dans cette indication qu'il était très efficace 
et bien toléré. L'efficacité de cette molécule a même été montrée comme supérieure aux corticoïdes chez les patients non répondants aux AINS (anti-inflammatoires non stéroïdiens) et/ou à la colchicine [73].

\section{Rilonacept}

Le rilonacept (Regeneron $\left.{ }^{\circledR}\right)$ était indiqué dans le traitement des CAPS. Cette protéine de fusion est composée d'un domaine Fc d'immunoglobuline IgG1 et de deux bras protéiques ce qui permet sa fixation à l'IL-1 $\beta$ et l'empêche ainsi de se fixer sur son récepteur. Une administration hebdomadaire par voie sous-cutanée était préconisée pour cette molécule. Ce médicament n'était pas disponible en France et a été retiré du marché dans les pays qui le commercialisait [69].

\section{Anakinra}

L'IL-1 Receptor antagonist (IL-1Ra) est l'antagoniste naturel du récepteur à l'IL-1. Cette glycoprotéine produite par les cellules myéloïdes, présente une faible homologie avec l'IL-1 $\beta$ et ne nécessite pas de maturation pour être secrétée [23]. L'IL-1Ra permet une régulation négative de l'effet de l'IL-1 $\beta$. Cet antagoniste agit en compétition avec l'IL-1 en se fixant aux récepteurs IL-1R1 avec une grande affinité et bloque la voie de signalisation proinflammatoire [74]. L'IL-1Ra régule ainsi les propriétés pro-inflammatoires en agissant comme un inhibiteur endogène de l'inflammation produite par l'IL-1 $\alpha$ et l'IL-1 $\beta$ [75]. En effet, des souris KO pour le gène de l'IL-1Ra ont développé spontanément des pathologies proches de la polyarthrite rhumatoïde [74].

L'IL-1Ra peut ainsi être utilisé en thérapeutique, sous une forme recombinante non glycosylée sous le nom d'anakinra (Kineret $\left.{ }^{\circledR}\right)$. Ce médicament a obtenu l'AMM en France dans le traitement de la polyarthrite rhumatoïde, en association avec le méthotrexate. Les patients atteints de CAPS sont également traités par l'anakinra à la posologie de 1 à 2 $\mathrm{mg} / \mathrm{kg} /$ jour par injection sous cutanée journalière. Ce traitement donne de très bons résultats cliniques et biologiques en bloquant la signalisation de l'IL-1 $\beta$ [76,77]. L'efficacité des thérapies anti-IL-1 $\beta$ a ainsi été démontrée chez les enfants atteints de NOMID/CINCA [78]. Cependant à l'heure actuelle, le Kineret ${ }^{\circledR}$ ne possède pas l'AMM en France dans le traitement des cryopyrinopathies. 
Les traitements anti-IL-1 sont très efficaces dans les pathologies impliquant l'inflammasome NLRP3 : les CAPS, les FMF résistantes à la colchicine, le syndrome de Schnitzler ou même les maladies auto-inflammatoires non classées $[60,64,65]$.

\section{Autres biothérapies}

D'autres biothérapies ont été développées comme des anticorps ciblant le récepteur à IL-6 (tocilizumab) ou à l'IL-18 (en cours de développement) et présentent un intérêt dans le traitement des maladies auto-inflammatoires [79]. Ainsi le tocilizumab peut être utilisé pour traiter la polyarthrite rhumatoïde, la maladie de Still de l'adulte ou le syndrome de Schnitzler chez des patients résistants aux traitements anti-IL-1 (anakinra et rilonacept) [80-81].

D'autres molécules ciblant l'inflammasome NLRP3 sont également en développement. En effet, une molécule de petite taille (MCC950), inhibiteur sélectif de l'activation de l'inflammasome NLRP3 a montré son efficacité pour traiter des souris développant des CAPS [82]. Le corps cétonique $\beta$-hydroxybutyrate a également été mis en évidence comme inhibiteur de l'inflammasome et donc de la production d'IL-1 $\beta$ dans différents modèles in vitro et in vivo [83]. Ceci offre donc des perspectives thérapeutiques dans le traitement des maladies auto-inflammatoires.

\section{Conclusion}

L'inflammasome est un complexe macromoléculaire intracellulaire permettant la libération de la cytokine pro-inflammatoire IL-1 $\beta$. Cette cytokine est impliquée dans de nombreuses pathologies inflammatoires comme les cryopyrinopathies, la polyarthrite rhumatoïde ou l'arthrite goutteuse. Ainsi une connaissance de l'inflammasome, son activation et des pathologies qui lui sont liées permet de mettre en place des thérapeutiques innovantes.

Déclaration de conflits d'intérêt : les auteurs déclarent ne pas avoir de conflits d'intérêts en relation avec cet article. 


\section{Références}

[1] Medzhitov R, Janeway CA Jr. Decoding the patterns of self and nonself by the innate immune system. Science 2002;296:298-300.

[2] Takeuchi O, Akira S. Pattern recognition receptors and inflammation. Cell 2010;140: 80520 .

[3] Imler JL, Hoffmann JA Toll receptors in innate immunity. Trends Cell Biol 2001;11:30411.

[4] Akira S, Uematsu S, Takeuchi O. Pathogen recognition and innate immunity. Cell 2006;124: 783-801.

[5] Takeda K, Akira S. Toll-like receptors in innate immunity. Int Immunol 2005;17:1-14.

[6] Beutler B, Rietschel ET. Innate immune sensing and its roots: the story of endotoxin. Nature Rev Immunol 2003;3:169-176.

[7] Ting JP, Lovering RC, Alnemri ES, Bertin J, Boss JM, Davis BK et al. The NLR gene family: a standard nomenclature. Immunity 2008;28:285-7.

[8] Saxena M, Yeretssian G. NOD-Like Receptors: Master Regulators of Inflammation and Cancer. Front Immunol 2014;5:327.

[9] Schroder K, Tschopp J. The inflammasomes. Cell 2010;140:821-32.

[10] Magalhaes JG, Sorbara MT, Girardin SE, Philpott DJ. What is new with Nods? Curr Opin Immunol 2011;23:29-34.

[11] Martinon F, Burns K, Tschopp J. The inflammasome: a molecular platform triggering activation of inflammatory caspases and processing of proIL-beta. Mol Cell 2002;10:417-26.

[12] Jamilloux Y, Henry T. The inflammasomes: platforms of innate immunity. Med Sci (Paris) 2013;29:975-84.

[13] Menu P, Vince JE. The NLRP3 inflammasome in health and disease: the good, the bad and the ugly. Clin Exp Immunol 2011;166:1-15.

[14] Guarda G, Zenger M, Yazdi AS, Schroder K, Ferrero I, Menu P et al. Differential expression of NLRP3 among hematopoietic cells. J Immunol 2011;186:2529-34.

[15] Dinarello CA. A signal for the caspase-1 inflammasome free of TLR. Immunity 2007;26:383-5.

[16] Cerretti DP, Kozlosky CJ, Mosley B, Nelson N, Van Ness K, Greenstreet TA et al. Science 1992;256:97-100.

[17] Thornberry NA, Bull HG, Calaycay JR, Chapman KT, Howard AD, Kostura MJ et al. A novel heterodimeric cysteine protease is required for interleukin-1 beta processing in monocytes. Nature 1992;356:768-74. 
[18] Schroder K, Zhou R, Tschopp J. The NLRP3 inflammasome: a sensor for metabolic danger? Science 2010;327:296-300.

[19] Denes A, Lopez-Castejon G, Brough D. Caspase-1: is IL-1 just the tip of the ICEberg ? Cell Death Dis 2012;3:e338.

[20] Dinarello CA. The IL-1 family and inflammatory diseases. Clin Exp Rheumatol 2000:20:S1-S13.

[21] Eleftheriadis T, Pissas G, Karioti A, Antoniadi G, Golfinopoulos S, Liakopoulos V et al. Uric acid induces caspase-1 activation, IL-1 $\beta$ secretion and P2X7 receptor dependent proliferation in primary human lymphocytes Hippokratia 2013;17:141-5.

[22] Feldmeyer L, Keller M, Niklaus G, Hohl D, Werner S, Beer HD. The inflammasome mediates UVB-induced activation and secretion of interleukin-1beta by keratinocytes. Curr Biol 2007; 17:1140-5.

[23] Garlanda C, Dinarello CA, Mantovani A. The interleukin-1 family: back to the future. Immunity 2013;39:1003-18.

[24] Sims JE, Smith DE. The IL-1 family: regulators of immunity. Nat Rev Immunol 2010;10:89-102.

[25] Martinon F, Mayor A, Tschopp J. The inflammasomes: guardians of the body. Annu Rev Immunol 2009;27:229-65.

[26] Sutterwala FS, Haasken S, Cassel SL. Mechanism of NLRP3 inflammasome activation. Ann NY Acad Sci 2014;1319:82-95.

[27] Pedra JH, Cassel SL, Sutterwala FS. Sensing pathogens and danger signals by the inflammasome. Curr Opin Immunol 2009;21:10-6.

[28] Eder C. Mechanisms of interleukin-1beta release. Immunobiology 2009;214:543-53.

[29] Rathinam VA, Jiang Z, Waggoner SN, Sharma S, Cole LE, Waggoner L et al. The AIM2 inflammasome is essential for host defense against cytosolic bacteria and DNA viruses. Nat Immunol 2010;11:395-402.

[30] Mariathasan S, Weiss DS, Newton K, McBride J, O'Rourke K et al. Cryopyrin activates the inflammasome in response to toxins and ATP. Nature 2006;440:228-32.

[31] Gicquel T, Victoni T, Fautrel A, Robert S, Gleonnec F, Guezingar M. Involvement of purinergic receptors and NLRP3-inflammasome pathway in the ATP-induced cytokine release from macrophages. Clin Exp Pharmacol Physiol 2014;41:279-86.

[32] Martinon F, Pétrilli V, Mayor A, Tardivel A, Tschopp J (2006) Gout-associated uric acid crystals activate the NALP3 inflammasome. Nature 440:237-41. 
[33] Gicquel T, Robert S, Loyer P, Victoni T, Bodin A, Ribault C et al. IL-1 $\beta$ production is dependent of the activation of purinergic receptors and NLRP3 pathway in human macrophages. FASEB J 2015;pii:fj.14-267393.

[34] Bauernfeind F, Ablasser A, Bartok E, Kim S, Schmid-Burgk J, Cavlar T et al. Inflammasomes: current understanding and open questions. Cell Mol Life Sci 2011;68:76583.

[35] Bauernfeind F, Bartok E, Rieger A, Franchi L, Núñez G, Hornung V. Cutting edge: reactive oxygen species inhibitors block priming, but not activation, of the NLRP3 inflammasome. J Immunol 2011;187:613-7.

[36] Gross O, Yazdi AS, Thomas CJ, Masin M, Heinz LX, Guarda G et al. Inflammasome activators induce interleukin-1a secretion via distinct pathways with differential requirement for the protease function of caspase-1. Immunity 2012;36:388-400.

[37] Halle A, Hornung V, Petzold GC, Stewart CR, Monks BG, Reinheckel T et al. The NALP3 inflammasome is involved in the innate immune response to amyloid-beta. Nat Immunol 2008;9:857-65.

[38] Dostert C, Pétrilli V, Van Bruggen R, Steele C, Mossman BT, Tschopp J. Innate immune activation through Nalp3 inflammasome sensing of asbestos and silica. Science 2008;320:674-77.

[39] Hornung V, Bauernfeind F, Halle A, Samstad EO, Kono H, Rock KL et al. Silica crystals and aluminum salts activate the NLRP3 inflammasome through phagosomal destabilization. Nat Immunol 2008;9:847-856.

[40] Cassel SL, Eisenbarth SC, Iyer SS, Sadler JJ, Colegio OR, Tephly LA et al. The Nalp3 inflammasome is essential for the development of silicosis. Proc Natl Acad Sci USA 2008;105:9035-9040.

[41] Yazdi AS, Guarda G, Riteau N, Drexler SK, Tardivel A, Couillin I et al. Nanoparticles activate the NLR pyrin domain containing 3 (Nlrp3) inflammasome and cause pulmonary inflammation through release of IL- $1 \alpha$ and IL-1 $\beta$. Proc Natl Acad Sci USA 2010;107:1944954.

[42] Martinon F. Signaling by ROS drives inflammasome activation. Eur J Immunol 2010;40:616-9.

[43] Pétrilli V, Papin S, Dostert C, Mayor A, Martinon F, Tschopp J. Activation of the NALP3 inflammasome is triggered by low intracellular potassium concentration. Cell Death Differ. 2007;14:1583-9.

[44] Ea HK. Mechanisms of gout inflammation. Press Med 2011;40:836-43.

[45] Martinon F. Mechanisms of uric acid crystal-mediated autoinflammation. Immunol Rev 2010;233:218-32. 
[46] Shi Y, Evans JE, Rock KL. Molecular identification of a danger signal that alerts the immune system to dying cells. Nature 2003;425:516-21.

[47] Gasse P, Riteau N, Charron S, Girre S, Fick L, Pétrilli V et al. Uric acid is a danger signal activating NALP3 inflammasome in lung injury inflammation and fibrosis. Am J Respir Crit Care Med 2009;179:903-13.

[48] Liu-Bryan R, Scott P, Sydlaske A, Rose DM, Terkeltaub R. Innate immunity conferred by Toll-like receptors 2 and 4 and myeloid differentiation factor 88 expression is pivotal to monosodium urate monohydrate crystal-induced inflammation. Arthritis Rheum 2005;52:2936-46.

[49] Scott P, Ma H, Viriyakosol S, Terkeltaub R, Liu-Bryan R. Engagement of CD14 mediates the inflammatory potential of monosodium urate crystals. J Immunol 2006;177:6370-8.

[50] Chen CJ, Shi Y, Hearn A, Fitzgerald K, Golenbock D, Reed G et al. MyD88dependent IL-1 receptor signaling is essential for gouty inflammation stimulated by monosodium urate crystals. J Clin Invest 2006;116: 2262-2271.

[51] Ng G, Sharma K, Ward SM, Desrosiers MD, Stephens LA, Schoel WM et al. Receptorindependent, direct membrane binding leads to cell-surface lipid sorting and Syk kinase activation in dendritic cells. Immunity 2008;29:807-18.

[52] Gombault A, Baron L, Couillin I. ATP release and purinergic signaling in NLRP3 inflammasome activation. Front Immunol 2013;3:414.

[53] Lee GS, Subramanian N, Kim AI, Aksentijevich I, Goldbach-Mansky R, Sacks DB et al. The calcium-sensing receptor regulates the NLRP3 inflammasome through $\mathrm{Ca} 2+$ and cAMP. Nature 2012;492:123-7.

[54] Villani AC, Lemire M, Fortin G, Louis E, Silverberg MS, Collette C et al. Common variants in the NLRP3 region contribute to Crohn's disease susceptibility. Nat Genet 2009;41:71-6.

[55] Jamilloux Y, Sève P, Henry T. Inflammasomes in human diseases. Rev Med Interne 2014;35:730-41.

[56] Rosengren S, Hoffman HM, Bugbee W, Boyle DL. Expression and regulation of cryopyrin and related proteins in rheumatoid arthritis synovium. Ann Rheum Dis 2005;64:708-14.

[57] Masters SL, Simon A, Aksentijevich I, Kastner DL. Horror autoinflammaticus : the molecular pathophysiology of autoinflammatory disease. Annu Rev Immunol 2009;27:62168.

[58] Boschan C, Witt O, Lohse P, Foeldvari I, Zappel H, Schweigerer L. Neonatal-onset multisystem inflammatory disease (NOMID) due to a novel S331R mutation of the CIAS1 
gene and response to interleukin-1 receptor antagonist treatment. Am J Med Genet A 2006 140:883-6.

[59] Agostini L, Martinon F, Burns K, McDermott MF, Hawkins PN, Tschopp J. NALP3 forms an IL-1beta-processing inflammasome with increased activity in Muckle-Wells autoinflammatory disorder. Immunity 2004;20:319-25.

[60] de Koning HD, Schalkwijk J, Stoffels M, Jongekrijg J, Jacobs JF, Verwiel E et al. The role of interleukin-1 beta in the pathophysiology of Schnitzler's syndrome. Arthritis Res Ther 2015;17:187.

[61] Stankovic Stojanovic K, Georgin-Lavialle S, Grateau G. Hereditary recurrent fever. Ann Dermatol Venereol 2014;141:538-45.

[62] Aksentijevich I, Putnam CD, Remmers EF, Mueller JL, Le J, Kolodner RD et al. The clinical continuum of cryopyrinopathies: novel CIAS1 mutations in North American patients and a new cryopyrin model. Arthritis Rheum 2007;56:1273-1285.

[63] Omenetti A, Carta S, Delfino L, Martini A, Gattorno M, Rubartelli A. Increased NLRP3dependent interleukin $1 \beta$ secretion in patients with familial Mediterranean fever: correlation with MEFV genotype. Ann Rheum Dis 2014 ;73:462-9.

[64] de Jesus AA, Canna SW, Liu Y, Goldbach-Mansky R. Molecular mechanisms in genetically defined autoinflammatory diseases: disorders of amplified danger signaling. Annu Rev Immunol 2015;33:823-74.

[65] Jéru I, Duquesnoy P, Fernandes-Alnemri T, Cochet E, Yu JW, Lackmy-Port-Lis M et al. Mutations in NALP12 cause hereditary periodic fever syndromes. Proc Natl Acad Sci U S A 2008;105:1614-9.

[66] Vance RE. The NAIP/NLRC4 inflammasomes. Curr Opin Immunol 2015;32:84-9.

[67] Kitamura A, Sasaki Y, Abe T, Kano H, Yasutomo K. An inherited mutation in NLRC4 causes autoinflammation in human and mice. J Exp Med 2014;211:2385-96.

[68] Church LD, McDermott MF. Canakinumab: a human anti-IL-1 $\beta$ monoclonal antibody for the treatment of cryopyrin-associated periodic syndromes. Expert Rev Clin Immunol 2010;6:831-41.

[69] Dubois EA, Rissmann R, Cohen AF. Rilonacept and canakinumab. Br J Clin Pharmacol 2011;71:639-41.

[70] Hoy SM. Canakinumab: a review of its use in the management of systemic juvenile idiopathic arthritis. BioDrugs 2015;2:133-42.

[71] Wulffraat NM, Woo P. Canakinumab in pediatric rheumatic diseases. Expert Opin Biol Ther 2013;13:615-22.

[72] Martin WJ, Walton M, Harper J. Resident macrophages initiating and driving inflammation in a monosodium urate monohydrate crystal-induced murine peritoneal model of acute gout. Arthritis Rheum 2009;60:281-9. 
[73] Hirsch JD, Gnanasakthy A, Lale R, Choi K, Sarkin AJ. Efficacy of Canakinumab vs. triamcinolone acetonide according to multiple gouty arthritis-related health outcomes measures. Int J Clin Pract 2014;68:1503-7.

[74] Arend WP, Gabay C. Physiologic role of interleukin-1 receptor antagonist. Arthritis Res $2000 ; 2: 245-8$.

[75] Dinarello CA. The role of the interleukin-1-receptor antagonist in blocking inflammation mediated by interleukin-1. N Engl J Med 2000;343:732-4.

[76] Chauffier K, London J, Beaudouin C, Fautrel B. Indications of anakinra. Press Med 2009;38:799-807.

[77] Hawkins PN, Lachmann HJ, Aganna E, McDermott MF. Spectrum of clinical features in Muckle-Wells syndrome and response to anakinra. Arthritis Rheum 2004;50:607-12.

[78] Goldbach-Mansky R, Dailey NJ, Canna SW, Gelabert A, Jones J, Rubin BI et al. Neonatal-onset multisystem inflammatory disease responsive to interleukin-1beta inhibition. N Engl J Med 2006;355:581-92.

[79] Li X, Zhang C, Chen W, Pan B, Kong F, Zheng K et al. Protective effect of neutralizing anti-IL-18 $\alpha$ monoclonal antibody on a mouse model of acute graft-versus-host disease. Oncol Rep 2015;34:2031-9.

[80] Nishina N, Kaneko Y, Kameda H, Takeuchi T. The effect of tocilizumab on preventing relapses in adult-onset Still's disease: A retrospective, single-center study. Mod Rheumatol $2015 ; 25: 401-4$.

[81] Krause K, Feist E, Fiene M, Kallinich T, Maurer M. Complete remission in 3 of 3 antiIL-6-treated patients with Schnitzler syndrome. J Allergy Clin Immunol 2012;129:848-50.

[82] Coll RC, Robertson AA, Chae JJ, Higgins SC, Muñoz-Planillo R, Inserra MC et al. A small-molecule inhibitor of the NLRP3 inflammasome for the treatment of inflammatory diseases. Nat Med 2015;21:248-55.

[83] Youm YH, Nguyen KY, Grant RW, Goldberg EL, Bodogai M, Kim D et al. The ketone metabolite $\beta$-hydroxybutyrate blocks NLRP3 inflammasome-mediated inflammatory disease. Nat Med 2015;21:263-9. 


\section{Figures}

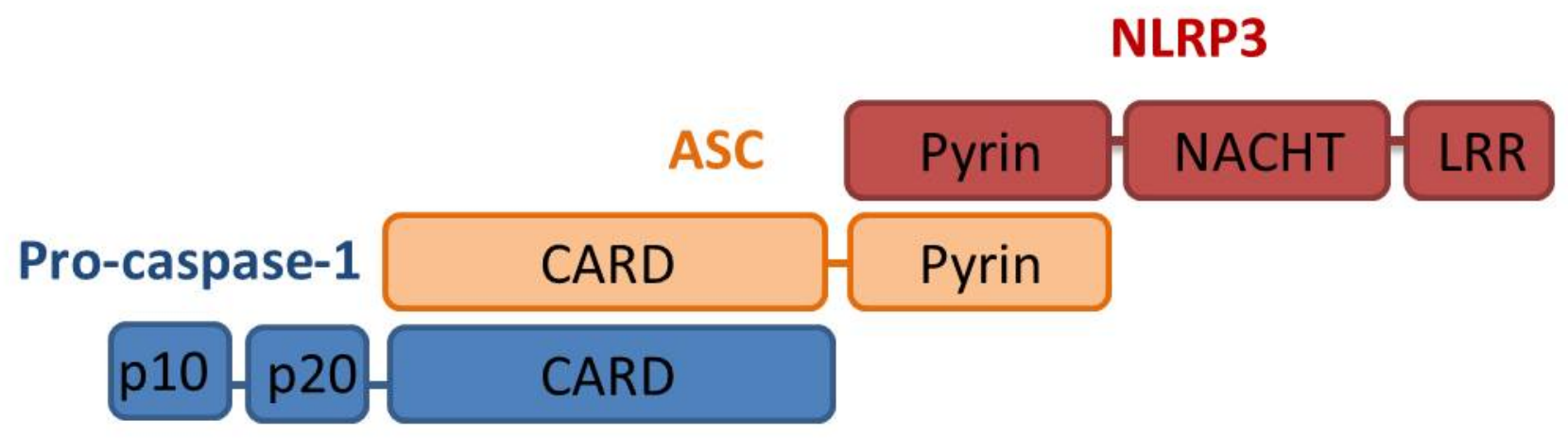

Figure 1. Structure de l'inflammasome NLRP3

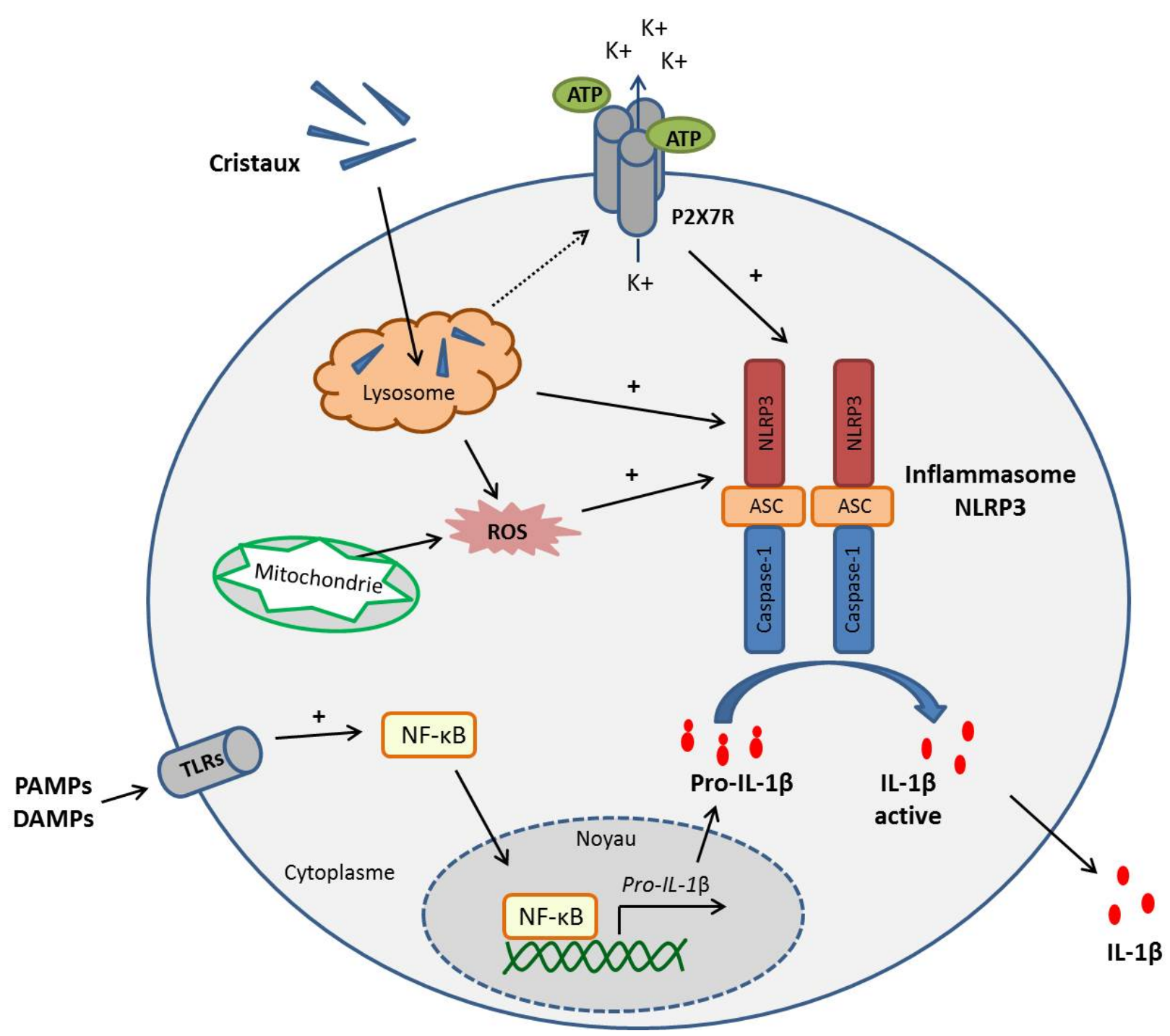

Figure 2. Modèle d'activation de l'inflammasome NLRP3 et libération d'IL-1 $\beta$ 


\section{Tableaux}

Tableau I.

Type d'activateur

Activateurs

Maladies associées

Candida albicans

Pathogènes

Staphylococcus aureus

Listeria monocytogenes

Infections

Virus Influenza

Toxine

Hémozoïne

Paludisme

Silice

Silicose

Origine environnementale

Amiante

Abestose

UV

Coup de soleil

ATP

Mort cellulaire

DAMPs

Glucose Diabète

Acide urique Arthrite goutteuse

Amyloïde $\beta \quad$ Maladie d'Alzheimer

Tableau I. Exemples d'activateurs de l'inflammasome NLRP3 et maladies associées 